\title{
Atomic processes modeling of X-ray free electron laser produced plasmas using SCFLY code
}

H.-K. Chung, B. I. Cho, O. Ciricosta, S. M. Vinko, J. S. Wark, and R. W. Lee

Citation: AIP Conference Proceedings 1811, 020001 (2017); doi: 10.1063/1.4975712

View online: $\mathrm{http} / / / \mathrm{dx}$.doi.org/10.1063/1.4975712

View Table of Contents: http://aip.scitation.org/toc/apc/1811/1

Published by the American Institute of Physics

\section{Articles you may be interested in}

Conference Photo: Atomic Processes in Plasmas (APiP 2016)

AIP Conference Proceedings 1811, 010002010002 (2017); 10.1063/1.4975710

Committees: Atomic Processes in Plasmas (APiP 2016)

AIP Conference Proceedings 1811, 010003010003 (2017); 10.1063/1.4975711

Preface: Atomic Processes in Plasmas (APiP 2016)

AIP Conference Proceedings 1811, 010001010001 (2017); 10.1063/1.4975709

The Tungsten Project: Dielectronic recombination data for collisional-radiative modelling in ITER AIP Conference Proceedings 1811, 030002030002 (2017); 10.1063/1.4975714 


\title{
Atomic processes modeling of X-ray free electron laser produced plasmas using SCFLY code
}

\author{
H.-K. Chung ${ }^{1 \text { a) }}$, B. I. Cho ${ }^{2}$, O. Ciricosta ${ }^{3}$, S. M. Vinko ${ }^{3}$, J. S. Wark ${ }^{3}$ and R. W. Lee ${ }^{4}$ \\ ${ }^{1}$ Nuclear Data Section, International Atomic Energy Agency, P.O. Box 100, A-1400, Vienna, Austria \\ ${ }^{2}$ Department of Physics and Photon Science, Gwangju Institute of Science and Technology, Gwangju 61005, \\ Korea \\ ${ }^{3}$ Department of Physics, Clarendon Laboratory, University of Oxford, Parks Road, Oxford OX1 3PU, UK \\ ${ }^{4}$ Institute for Material Dynamics at Extreme Conditions, University of California, Berkeley, CA 94720, USA \\ a)h.chung@iaea.org
}

\begin{abstract}
With the development of X-ray free electron lasers (XFEL), a novel state of matter of highly transient and non-equilibrium plasma has been created in laboratories. As high intensity X-ray laser beams interact with a solid density target, electrons are ionized from inner-shell orbitals and these electrons and XFEL photons create dense and finite temperature plasmas. In order to study atomic processes in XFEL driven plasmas, the atomic kinetics model SCFLY containing an extensive set of configurations needed for solid density plasmas was applied to study atomic processes of XFEL driven systems. The code accepts the time-dependent conditions of the XFEL as input parameters, and computes time-dependent population distributions and ionization distributions self-consistently with electron temperatures and densities assuming an instantaneous equilibration of electron energies. The methods and assumptions in the atomic kinetics model and unique aspects of atomic processes in XFEL driven plasmas are described.
\end{abstract}

\section{INTRODUCTION}

The Linear Collider Light Source (LCLS) X-ray free electron laser at SLAC National Laboratory can provide $\sim 10^{12}$ photons with energies of a few keVs in sub picosecond pulses [1,2]. This short pulse of photons of high energy interacts mainly with the bound electrons of atoms in the sample, preferentially photoionizing the inner-shell electrons leaving atoms in highly transient states. The transient atomic states with inner-shell holes try to stabilize by transitions to the lower energy states by either Auger decay or photon emission with a time scale comparable to the XFEL pulse-length. The transient atomic states may be further ionized by high intensity photons resulting in so-called "hollow atoms" that have two holes in the K-shell [1]. Depending on the plasma density, the Auger and photoionized electrons may participate in collisions with atoms and ions in the system and ionize them further [3]. The stabilizing photon emission from the transient states via absorption or scattering of X-ray photons have been used as diagnostics to obtain detailed information on the atomic state evolution of the matter $[2,3,4]$.

Spectroscopic measurements are interpreted with a population kinetics model, which includes relevant atomic processes such as photo absorption and emission, Auger decay and radiative stabilization as well as electron collisions. Atomic states relevant to these processes such as double K-hole states created by X-ray photons and population decay channels such as the Auger process and radiative stabilization should be considered. When a target is near-solid density, one should consider an extensive set of atomic levels in order to describe the near-LTE states [5] as collisions by electrons are dominant and atomic level population approach to the thermodynamic equilibrium represented by statistical distributions given by the local plasma conditions. Most importantly, the atomic state distributions should be treated in a time-dependent fashion as the time scale of atomic processes involving highly transient states is comparable to the length of the driving X-ray pulse, on the order of tens of femtoseconds. As soon as the driving photon pulse is over, transient state populations and their associated photon emission quickly disappear, and atomic states reach a pseudo-steady plasma state at the end of the pulse [6].

Recently, Collisional-Radiative (CR) models employing super-configuration atomic levels have been successfully used for a wide range of plasma conditions where electron collisional processes are prevalent [7]. Particularly, a generalized CR model using hydrogenic configurations, FLYCHK [8], provided experimentalists

Atomic Processes in Plasmas APiP 2016

AIP Conf. Proc. 1811, 020001-1-020001-7; doi: 10.1063/1.4975712

Published by AIP Publishing. 978-0-7354-1479-2/\$30.00 
with fast and simple but reasonable predictions of atomic properties of plasmas. In order to study XFEL driven plasmas, the FLYCHK code was extended to the atomic kinetics model SCFLY containing an extensive set of configurations needed for solid density plasmas [5]. The code accepts the time-dependent conditions of the XFEL as input parameters, and computes time-dependent population and ionization distributions selfconsistently with electron temperatures and densities assuming an instantaneous electron-electron equilibration. The methods and assumptions in the atomic kinetics model and applications to XFEL experiments are described here.

\section{METHODS}

XFEL photons create an exotic state of matter for studies of high energy density physics. High energy photons heat the target volumetrically and generate quasi-uniform plasmas, which have little hydrodynamic motion on the time scale of the XFEL pulse, and hence spatial gradients are minimized during the pulse. The high photon number enables a single shot experiment with a high repetition rate due to high signal-to-noise ratios [2]

From the point of view of atomic processes, XFEL photons create ionized states with photo-ionization processes. In particular, K-shell hole states which subsequently ionize to the next charge state by Auger decay. Primarily, photo electrons, created by photoionization events, and Auger electrons, created by subsequent Auger processes, lead to a non-equilibrium distribution of the electron energy density function that has high energy tails. When the XFEL interacts with a solid density target, photo electrons and Auger electrons ionize the solid target and secondary electrons are quickly produced with much lower energies than the primary electrons. With a substantial amount of ionization, secondary electrons quickly establish a Maxwellian distribution by collisions, at a temperature which can be estimated from the photon energy absorbed in the target and the internal energy of the dense plasma. Therefore, population distributions, photon absorption rates, internal energies and average charge states should be considered in a time-dependent fashion and self-consistently in order to obtain time-dependent temperature and density conditions for the XFEL heated dense matter.

\section{Population Kinetics Model}

The method chosen to study atomic processes in plasmas is to solve rate equations linking atomic states via atomic transitions. A population kinetics model sets up a grid of atomic states in an atom or ion and solves for a population distribution by considering population and depopulation rates due to various atomic processes. The population of the $i^{\text {th }}$ state $n_{i}$ is obtained from the following rate equations where $W_{i j}$ refers to a transition from the $i^{\text {th }}$ state to $j^{\text {th }}$ state.

$$
\frac{d n_{i}}{d t}=-n_{i} \prod_{j \neq i}^{N_{\max }} W_{i j}+\prod_{j \neq i}^{N_{\max }} n_{j} W_{j i}
$$

A matrix form of the set of rate equations is solved for a population matrix:

$$
\frac{d \tilde{n}}{d t}=\bar{W} \cdot \tilde{n}
$$

The included atomic transitions are electron collisional excitation and deexcitation, spontaneous decay, photoexcitation and stimulated decay within a charge state and electron collisional ionization and recombination, photoionization and radiative recombination, autoionization and electron capture between different charge states. Note that the summation of transitions is limited by $N_{\max }$. As an ion is surrounded by electrons, photons and other ions, the number of transitions needed to determine the population distributions is finite. The cut-off limit is often obtained by applying the model of Stewart and Pyatt $[9,10]$ where the high-lying states are assumed to disappear due to interactions with surrounding electrons and ions so that the ionization potential required to ionize a bound electron is reduced. The reduction of ionization potential, i.e., so called ionization potential depression (IPD) plays a significant role in determining partition functions of atomic states of high density plasmas. The validity of this model has been challenged recently, where experimental data shows it to be in error at conditions produced at LCLS $[3,11]$ but in better agreement with sophisticated density functional theory calculations [12].

Rate coefficients require information on plasma density and temperature at a given time. Time-dependent electron densities are computed by invoking the condition of charge neutrality at each time step. As we are interested in atomic processes taking place during an X-ray pulse shorter than a pico-second, the plasma is 
assumed to be stationary. Time-dependent electron temperatures are computed by an energy balance equation where absorbed photon energy is equal to the sum of internal energy and plasma thermal energy. The photon energy absorbed by the target is assumed to be transferred to electron thermal energy with no energy transferred to ion motion. Then the electron temperature and density are computed self-consistently providing a population distribution as a function time given an input description of the XFEL pulse.

\section{Atomic Data}

The accuracy of a model is often viewed as dependent on the accuracy of atomic data. For many plasma applications, however, the completeness of atomic states and transitions contained in a model makes more significant impact in accuracy [13]. Particularly, as an electron density increases, excited states and autoionization states play as significant role as excitation, ionization or recombination channels and it is extremely important to have a converged set of atomic states and transitions [5]. If one wishes to use more accurate descriptions of atomic states, for example, fine-structure levels or term states, the model becomes so large that it is nearly impossible to solve the rate equations even with the greatest computer resources accessible today. In the case of XFEL produced solid density plasmas, the number of atomic states can be extremely large due to autoionizing states such as two electron K-hole states created by the intense XFEL photons. If one constructs a model using term states or fine-structure levels and limits the calculation to a few configurations, it may not count all the relevant atomic processes and the results may not be convergent. The other problem with a large model is the difficulty in obtaining time-dependent solutions, which is essential to study XFEL plasmas.

For a tractable model of XFEL driven plasmas, we employ screened-hydrogenic configurations, a type of super-configurations. At finite densities, relative population distributions among closely-lying levels can be represented by statistical distributions, for example, among Rydberg states close to the continuum limit, or among fine-structure or term states belonging to a configuration. A screened-hydrogenic model exploits the statistical distribution among fine-structure or term states and represents an atomic state by the principal quantum number of each bound electron. It is noted that a great attention was paid to the accuracy of atomic data and the simple screened hydrogenic data used in the FLYCHK code was replaced with calculated and averaged data in a Dirac-Hartree-Slater approximation [14] in the SCFLY code applied to XFEL problems [15]. More details on a screened hydrogenic model and detailed atomic data used in the SCFLY code can be found in the ref. $[7,8,15]$.

\section{RESULTS}

A collisional-radiative model employing screened hydrogenic levels has been successfully applied to describe spectroscopic signatures of a series of XFEL experiments [2-4, 11, 15-17]. Results show that observed spectra depend on the incoming XFEL photon energy, intensity and total energy of the pulse in a nonlinear manner as the photons interact with XFEL heated plasma. In this section, we investigate interesting features of atomic processes in solid density plasmas interacting with XFEL photons.

First, the absorption mechanism of photons interacting with solid density target is complex since the absorption is not only time-dependent but also nonlinear as demonstrated in Figure 1. It illustrates the timedependent photon absorption processes with opacity calculations for $1588 \mathrm{eV}$ photons ionizing a solid density aluminum target. If the energy of incoming photons $\mathrm{E}_{\mathrm{p}}$, is not tuned to any of the bound-bound transitions of the initial target (neutral state), the absorption process is driven by bound-free photoionization processes where outer-shell and/or inner-shell electrons are ionized by photons depending on the ionization potential of the ion and photon energy $[4,16]$. That is, if $\mathrm{E}_{\mathrm{p}}$ is greater than the K-edge, photons will primarily create $\mathrm{K}$-shell holes, and if not, photons will ionize only outer-shell electrons. Photo electrons produced by the XFEL pulse and Auger electrons from autoionizing states, which are called primary electrons in this paper, will increase in number and interact with the plasma by ionization and excitation.

If the XFEL photon energy is tuned to resonant $\mathrm{K}_{\alpha}$ transition energies of an ion lower than the K-edge energy, the absorption process starts with photoionization of outer shell ions of low charged ions starting from neutral. As soon as the ion is populated, the absorption process becomes dominated by $\mathrm{K}_{\alpha}$ absorption resonant to $\mathrm{E}_{\mathrm{p}}$. In this case, the corresponding $\mathrm{K}_{\alpha}$ spectra of the particular ion are observed to be much brighter than those of other ions [4]. The resonant absorption of incoming photon energy by $\mathrm{K}_{\alpha}$ transitions turns on and off as ionization progresses and hence the opacity changes drastically as the charge state distribution changes with ionization [16]. Free-free absorption processes always exist, but are many orders smaller in magnitude compared with bound-bound or bound-free processes, unless the electron density is very high. As the absorption process of XFEL photons depends on both bound-free and bound-bound processes as time and ionization 
progresses, the total absorption of XFEL photons and observed spectra are heavily influenced by the rate of absorption, ionization, and most importantly the XFEL photon energy and intensity.

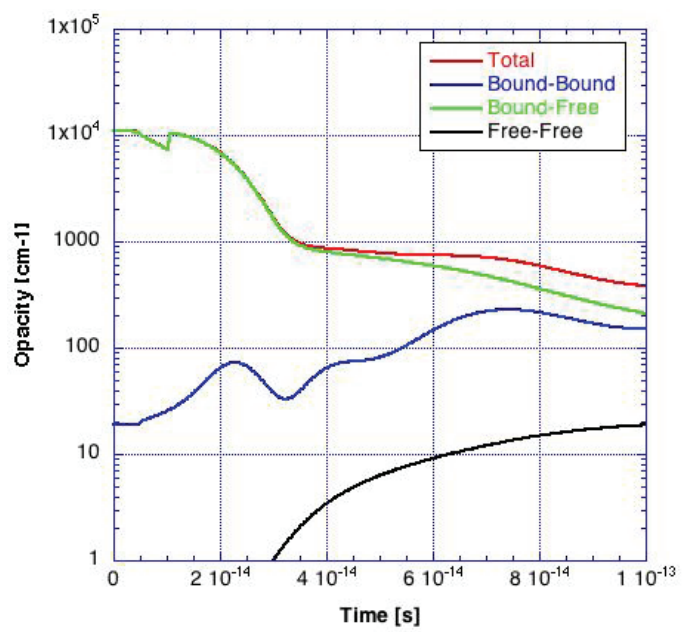

FIGURE 1. Total, bound-bound, bound-free and free-free opacities of incoming XFEL photons of $1588 \mathrm{eV}$ are plotted as a function of time for solid-density aluminum target. As the XFEL photons have an energy greater than the K-edge energy of $1559.6 \mathrm{eV}$, the photo-ionization processes, i.e., the bound-free contribution, dominate early in the pulse.

The bound-bound absorption must be considered with care within a simulation of the XFEL problem because the X-ray intensity is 10 orders of magnitude higher than conventional X-ray sources. Generally, bound-bound absorption processes are considered with a delta function since the spectral line shapes of $\mathrm{K}_{\alpha}$ transitions are very narrow and generally only influenced by Doppler and Natural broadening. As illustrated in Figure 2, the transition at $1487 \mathrm{eV}$ has an absorption strength 400 times lower at $1492 \mathrm{eV}$ for neutral aluminum. For $1492 \mathrm{eV}$ XFEL photons, the photoexcitation rate coefficient is zero in a delta function approximation since the photo excitation rate coefficient is 400 times smaller than the resonant energy at $1487 \mathrm{eV}$. However, as the XFEL photon intensity is many orders of magnitude larger than any other X-ray sources, the resultant photoexcitation at $1492 \mathrm{eV}$ could be sufficiently substantial to influence population distributions.

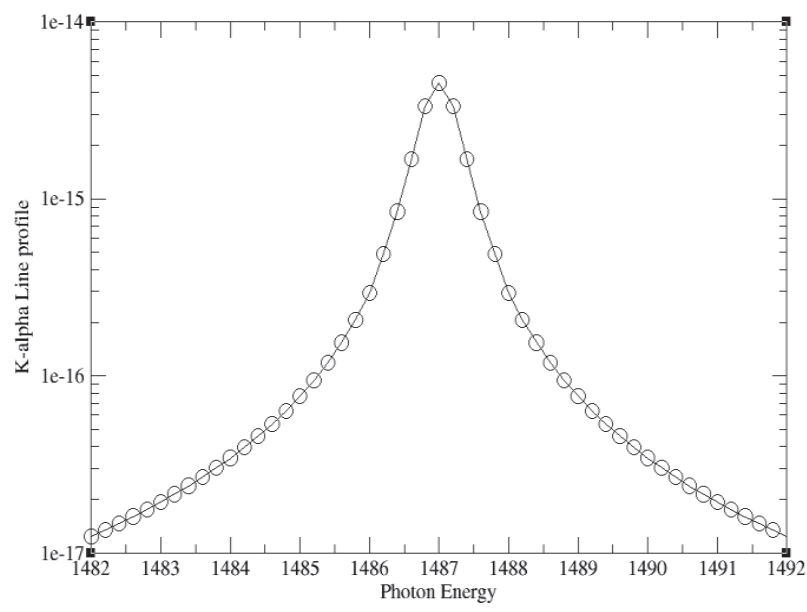

FIGURE 2. Spectral line shapes of the $\mathrm{K}_{\alpha}$ transition at $1487 \mathrm{eV}$ of Aluminum target broadened by Natural broadening mechanisms.

The energy distribution of primary electrons, that is, photo electrons from photoionization and Auger electrons from inner-shell hole states, is critical in determining the evolution of population distributions and 
plasma conditions. Since the target is at solid density, the electrons have a high probability of having collisions within the target, and a large number of low energy secondary electrons are produced by collisional ionization processes. Secondary electrons build up quickly and their energy distribution is close to a Maxwellian distribution, while primary electrons continue to be produced at discrete high energies [18]. The time scale of establishing a Maxwellian distribution depends on incoming photon conditions and target density. A code $\mathrm{Ct} 27$ was developed to calculate time-dependent electron energy distributions by solving the Boltzmann equations coupled to the population kinetics model FLYCHK. It includes elastic and inelastic collisions as well as sources and sinks produced by ionization and recombination, respectively, under the assumption of zero dimension [19]. Preliminary results of time-dependent electron energy distributions are shown in Figure 3 for $10^{12}$ photons in a $200 \mathrm{fs}$ pulse having an energy of $100 \mathrm{eV}$, impinging on a solid aluminum foil with a focal spot of 40 microns diameter. The VUV photons initially create primary electrons at discrete energies, and subsequently thermal electrons establish a Maxwellian distribution after $10 \mathrm{fs}$. In this example, energies of two electrons created by an ionization event are assumed to have a probability of constant distribution over a certain energy range. Other possible assumptions include a delta function at zero energy or a more realistic distribution, which establish the Maxwellian distribution sooner than the constant distribution assumption, but, they have not been implemented yet.

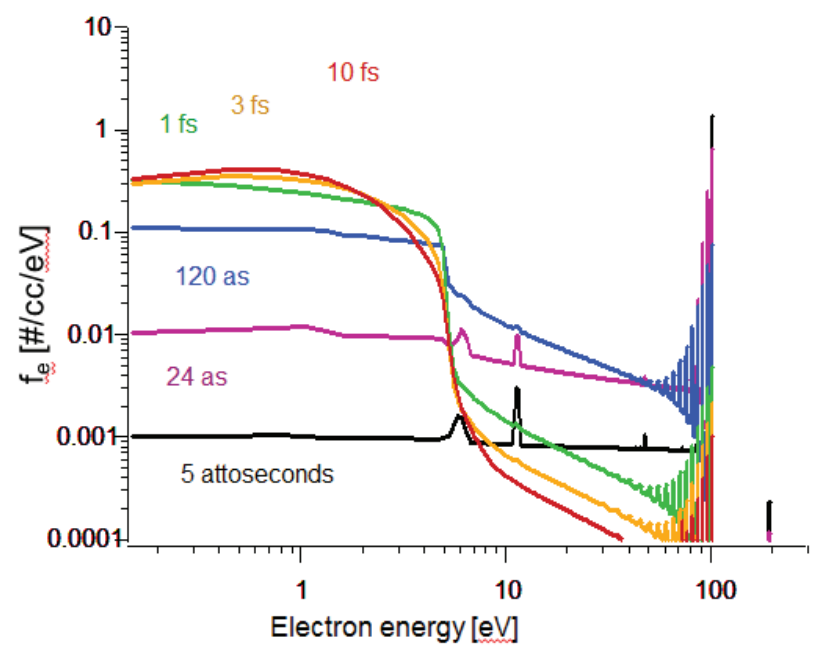

FIGURE 3. A time-dependent electron energy distribution as a function of time and electron energy for an example of 200 $\mathrm{fs}, 200 \mathrm{eV}, 10^{12}$ photons ionizing solid density aluminum target of 40 micron spot.

Time-dependent average temperatures are compared in Figure 4 between the results of the $\mathrm{Ct} 27$ code and the result of the SCFLY code where the photon energy absorbed in a target is assumed to be instantaneously divided between electron thermal energy and internal energy. Due to the instantaneous energy distribution assumption, the electron temperature calculated by SCFLY code is smaller early in time and the high temperatures in the beginning are due to primary electrons and very few thermal electrons. Later it compares well with the value of $\mathrm{Ct} 27$ code as electrons thermalize to a Maxwellian distribution and two results are comparable already by 2-3 fs, which largely justifies the use of the instantaneous thermalization assumption for population kinetics calculations. For a solid density target, the thermal electrons produced by photons participate in the ionization processes, and electron collisions dominate over photoionization processes at later times [2]. However, the instantaneous thermalization assumption may not be valid for very high energy photons and low density targets where it takes a much longer time for the electron energy distributions to come into equilibrium.

Finally, we would like to emphasize that the ionization potential depression (IPD) is a critical factor in modeling atomic processes and observed spectra for solid density targets. The reduction of the ionization potential and inability to populate high-lying states drastically change the population distribution and charge state distribution by altering transition rates, dominant population channels and partition functions of atomic states. This sensitivity of population distributions to the IPD has been observed in a series of XFEL experiments by spectroscopic methods. $[3,11]$ 


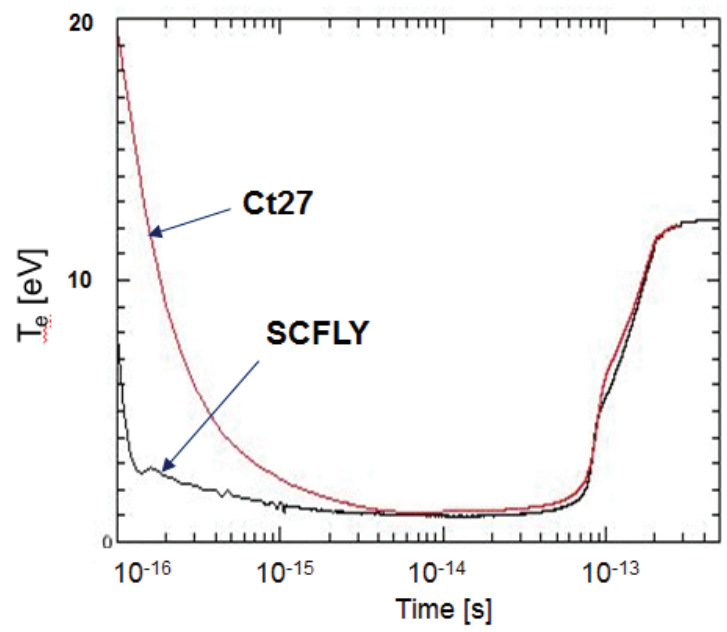

FIGURE 4. A time-dependent electron temperature is compared between Ct27 code and SCFLY code results for an example of $200 \mathrm{fs}, 200 \mathrm{eV}, 10^{12}$ photons ionizing solid density aluminum target of 40 micron spot.

\section{SUMMARY}

Observed spectra of XFEL experiments have been successfully studied with a collisional-radiative model using screened-hydrogenic levels. It is shown that absorption processes of XFEL photons are nonlinear and complex. Photon absorption and population evolution should be self-consistently treated to understand the evolution of plasma conditions. Special care is needed in implementing bound-bound absorption processes and ionization potential depression.

Though the SCFLY code using the screened-hydrogenic model has been successfully applied to many XFEL problems, the application is rather limited to spatially uniform, simple cases where 1) hydrogenic assumptions are valid as $\Delta \mathrm{n}=0$ transitions are not important, 2) the initial solid-state conditions do not have a significant impact in the evolution of population distributions and 3) the electron energy distribution function is close to Maxwellian. For more complex systems, there exist other theoretical approaches using more detailed atomic structures, or solving spatial distributions and non-equilibrium electron energy distribution functions selfconsistently with population kinetics [20-24].

\section{ACKNOWLEDGMENTS}

J.S.W. and O.C are grateful to EPSRC for support under grant number EP/L000849/1. B.I.C. was supported by the NRF of Korea (NRF-2016R1A2B4009631). S.M.V. gratefully acknowledges support from the Royal Society.

\section{REFERENCES}

1. L. Young, E. P. Kanter, B. Krässig, Y. Li, A. M. March, S. T. Pratt, R. Santra, S. H. Southworth, N. Rohringer, L. F. DiMauro, G. Doumy, C. A. Roedig, N. Berrah, L. Fang, M. Hoener, P. H. Bucksbaum, J. P. Cryan, S. Ghimire, J. M. Glownia, D. A. Reis, J. D. Bozek, C. Bostedt, and M. Messerschmidt, Nature 466, 56 (2010).

2. S. M. Vinko, O. Ciricosta, B. I. Cho, K. Engelhorn, H.-K. Chung, C. R. D. Brown, T. Burian, J, Chalupský, R. W. Falcone, C. Graves, V. Hajkova, A. Higginbotham, L. Juha, J. Krzywinski, H. J. Lee, M. Messerschmidt, C. D. Murphy, Y. Ping, A. Scherz, W. Schlotter, S. Toleikis, J. J. Turner, L. Vysin, T. Wang, B. Wu, U. Zastrau, D. Zhu, R. W. Lee, P. A. Heimann, B. Nagler, and J. S. Wark, Nature 482, 59 (2012).

3. O. Ciricosta, S. M. Vinko, H.-K. Chung, B.-I. Cho, C. R. D. Brown, T. Burian, J. Chalupský, K. Engelhorn, R. W. Falcone, C. Graves, V.Hajkova, A. Higginbotham, L. Juha, J. Krzywinski, H. J. Lee, M. 
Messerschmidt, C. D. Murphy, Y. Ping, D. S. Rackstraw, A. Scherz, W. Schlotter, S. Toleikis, J. J. Turner, L. Vysin, T. Wang, B. Wu, U. Zastrau, D. Zhu, R. W. Lee, P. Heimann, B. Nagler, and J. S. Wark, Phys. Rev. Lett. 109, 065002 (2012).

4. B. I. Cho, K. Engelhorn, S. M. Vinko, H.-K. Chung, O. Ciricosta, D. S. Rackstraw, R. W. Falcone, C. R. D. Brown, T. Burian, J. Chalupský, C. Graves, V. H_ajkov_a, A. Higginbotham, L. Juha, J. Krzywinski, H. J. Lee, M. Messersmidt, C. Murphy, Y. Ping, N. Rohringer, A. Scherz, W. Schlotter, S. Toleikis, J. J. Turner, L. Vysin, T. Wang, B. Wu, U. Zastrau, D. Zhu, R. W. Lee, B. Nagler, J. S. Wark, and P. A. Heimann, Phys. Rev. Lett. 109, 245003 (2012).

5. H.-K. Chung, M. Chen, and R. Lee, High Energy Density Phys. 3, 57 (2007).

6. H.-K. Chung and R.W. Lee, High Energy Density Physics, 5, 1 (2009) .

7. H.-K. Chung, S. H. Hansen, H. A. Scott, "Generalized Collisional Radiative Model Using Screened Hydrogenic Levels" in Modern Methods in Collisional-Radiative Modeling of Plasmas, edited by Yu. Ralchenko (Springer, 2016) pp.51-79.

8. H.-K. Chung, M. Chen, W. Morgan, Y. Ralchenko, and R. Lee, High Energy Density Phys. 1, 3 (2005).

9. J. C. Stewart and K. D. Pyatt, Jr., Astrophys. J. 144, 1203 (1966).

10. D. J. Hoarty, P. Allan, S. F. James, C. R. D. Brown, L. M. R. Hobbs, M. P. Hill, J. W. O. Harris, J. Morton, M. G. Brookes, R. Shepherd, J. Dunn, H. Chen, E. Von Marley, P. Beiersdorfer, H. K. Chung, R. W. Lee, G. Brown, and J. Emig, Phys. Rev. Lett. 110, 265003 (2013).

11. O. Ciricosta, S. M. Vinko, B. Barbrel, D. S. Rackstraw, T. R. Preston, T. Burian, J. Chalupský, B. I. Cho, H. -K. Chung, G. L. Dakovski, K. Engelhorn, V. Hájková, P. Heimann, M. Holmes, L. Juha, J. Krzywinski, R. W. Lee, S. Toleikis, J. J. Turner, U. Zastrau, J. S. Wark, Nature Communications 7, 11713 (2016).

12. S. M. Vinko, O. Ciricosta, J. S. Wark, Nature Communications, 5, 3533 (2014)

13. S. B. Hansen, "Balancing Detail and Completeness in Collisional-Radiative Models" in Modern Methods in Collisional-Radiative Modeling of Plasmas, edited by Yu. Ralchenko (Springer, 2016) pp.1-15.

14. M.H. Chen, E. Laiman, B. Casemann, M. Aoyagi, H. Mark, Phys. Rev. A, 19, 2253 (1979)

15. O. Ciricosta, S. M. Vinko, H.-K. Chung, C. Jackson, R. W. Lee, T. R. Preston, D. S. Rackstraw, J. S. Wark, Phys. Plasmas, 23, 022707 (2016)

16. D. S. Rackstraw, O. Ciricosta, S. M. Vinko, B. Barbrel, T. Burian, J. Chalupský, B. I. Cho, H.-K. Chung, G. L. Dakovski, K. Engelhorn, V. Hajkova, P. Heimann, M. Holmes, L. Juha, J. Krzywinski, R. W. Lee, S. Toleikis, J. J. Turner, U. Zastrau, and J. S. Wark, Phys. Rev. Lett. 114, 015003 (2015).

17. S. M. Vinko, O. Ciricosta, T. R. Preston, D. S. Rackstraw, C.R.D. Brown, T. Burian, J. Chalupský, B. I. Cho, H.-K. Chung, K. Engelhorn, R. W. Falcone, R. Fiokovinini, V. Hájková, P. A. Heimann, L. Juha, H. J. Lee, R. W. Lee, M. Messerschmidt, B. Nagler, W. Schlotter, J. J. Turner, L. Vysin, U. Zastrau, J. S. Wark, Nature Communications 6, 6397 (2015).

18. N. Medvedev, U. Zastrau, E. Förster, D. O. Gericke, and B. Rethfeld, Phys. Rev. Lett. 107, 165003 (2011).

19. H.-K. Chung, W. L. Morgan, R. W. Lee, Proceedings of the Third International Conference on Inertial Fusion Sciences and Applications, p303 (2004).

20. O. Peyrusse, "Collisional-Radiative Modeling and Interaction of Monochromatic X-Rays with Matter" in Modern Methods in Collisional-Radiative Modeling of Plasmas, edited by Yu. Ralchenko (Springer, 2016) pp.127-151

21. S. P. Hau-Riege, R. A. London, and A. Szoke, Phys. Rev. E 69, 051906 (2004)

22. O. Peyrusse, Phys. Rev. E 86, 036403 (2012)

23. Z. Jurek, S.-K. Son, B. Ziaja, R. Santra, J. App. Cryst. 49 (2016)

24. B. Ziaja, V. Saxena, S.-K. Son, N. Medvedev, B. Barbrel, B. Woloncewicz, M. Stranky, Phys. Rev. E 93 , 053210 (2016) 